\title{
Tsafon
}

Revue d'études juives du Nord

$72 \mid 2016$

Juifs, Israéliens, dans la littérature française et israélienne

\section{Le sauvetage des juifs au Chambon-sur-Lignon à travers Les Cerfs-volants de Romain Gary}

Ingrid Letourneau

\section{(2)enEdition}

\section{Journals}

Édition électronique

URL : https://journals.openedition.org/tsafon/358

DOI : $10.4000 /$ tsafon.358

ISSN : 2609-6420

Éditeur

Association Jean-Marie Delmaire

Édition imprimée

Date de publication : 1 décembre 2016

Pagination : 35-46

ISSN : $1149-6630$

Référence électronique

Ingrid Letourneau, «Le sauvetage des juifs au Chambon-sur-Lignon à travers Les Cerfs-volants de Romain Gary », Tsafon [En ligne], 72 | 2016, mis en ligne le 31 mai 2018, consulté le 25 juin 2021. URL: http://journals.openedition.org/tsafon/358 ; DOI : https://doi.org/10.4000/tsafon.358 


\title{
Le sauvetage des juifs au Chambon-sur-Lignon à travers Les Cerfs-volants de Romain Gary
}

\author{
Ingrid Letourneau*
}

En 1977, Romain Gary, qui fut l'un des premiers à rejoindre la Résistance du général de Gaulle auprès des Forces Françaises libres, accepte la proposition de l'Ordre de la Chancellerie de travailler à un documentaire sur les Compagnons de la Libération. Il renonce cependant bientôt à poursuivre ce projet, soulignant la difficulté qui se présente à lui d'éviter l'écueil de cette « injustice qui consisterait dans le choix des uns et l'ignorance des autres ${ }^{1}$ ainsi que les lacunes de la forme documentaire qui ne lui permettrait pas de restituer toute «l'épaisseur humaine $»^{2}$ de ses anciens camarades.

\section{Le Chambon-sur-Lignon,} un nom qui résonne au sein de la fiction garyenne

Pourtant, Gary n'en a pas encore fini avec la Résistance. En 1980, trente-cinq ans après Éducation européenne, son premier roman sur la Résistance (1945), paraît Les Cerfs-volants. Cette œuvre, l'ultime de

\footnotetext{
*Université Charles de Gaulle - Lille 3.

${ }^{1}$ Copie d'une lettre du 17 novembre 1978 adressée par Romain Gary à Jean Claude Lattès. Correspondance d'édition Romain Gary, archives Gallimard. Retranscrite par Fabrice Larat dans Romain Gary : un itinéraire européen, Chêne-Bourg, Georg Éditeur, coll. « Europe », 1999, p. 54.

${ }^{2}$ Romain Gary, Archives du musée de l'Ordre de la Libération, dossier personnel de Romain Gary, note tapuscrite, non datée, citée par Kerwin Spire dans son article «Romain Gary, des Compagnons de la Libération aux Justes parmi les Nations », Europe, $\mathrm{n}^{\circ} 1022-1023$, juin-juillet 2014, p. 77.
} 
l'auteur, adopte la forme du témoignage fictif de Ludo, personnage atteint d'un « excès de mémoire ${ }^{3}$ qui raconte, des dizaines d'années après, sa jeunesse résistante à Cléry, un petit village normand inventé par Gary. Au sein de cet univers fictif, résonne néanmoins le nom d'un village bien réel : celui du Chambon-sur-Lignon, évoqué à travers l'engagement d'Ambroise Fleury, l'oncle de Ludo, qui s'y rend pour aider les villageois dans le sauvetage des juifs et apporter un peu de douceur aux réfugiés avec les cerfs-volants qu'il fabrique.

Le Chambon-sur-Lignon, «village de huguenots» (CV, 318) comme le décrit Souba, camarade de maquis du narrateur, commence à être bien connu dans les années soixante-dix pour avoir assuré l'accueil, la protection et le sauvetage de réfugiés face aux menaces d'arrestation et de déportation. Une bonne majorité de ces réfugiés était des enfants juifs ${ }^{4}$, mais le village accueillait aussi des « opposants politiques au régime nazi et à l'État français collaborationniste $»^{5}$. L'ensemble des Chambonnais a ainsi pris part à cette forme de résistance civile. Certains, tel Fleury qui se « fait pincer entre Lyon et la frontière suisse » $(\mathrm{CV}, 318)$, organisent l'évacuation des réfugiés vers la Suisse. D'autres font office de faussaire, tandis que beaucoup accueillent des enfants juifs dans leur ferme, sous le sceau du secret. L'ampleur de cette résistance et le grand nombre de juifs sauvés font bien de ce village «l'un des symboles du refuge pour les persécutés ${ }^{6}$ sous le régime de Vichy.

À la fin des années soixante-dix, Gary a pu entendre parler du Chambon-sur-Lignon par de nombreux biais: à l'occasion de la nomination d'André et de Magda Trocmé comme Justes parmi les Nations par l'institut Yad Vashem d'Israël en 1971, de l'inauguration de la plaque commémorative en hommage aux habitants du Chambon-surLignon le 17 juin 1979 au nom des « Juifs réfugiés au Chambon-surLignon et dans les communes avoisinantes », de la parution du Sang des

\footnotetext{
${ }^{3}$ Les Cerfs-volants [1980], Paris, Gallimard, coll. « folio », 1983, p. 26. La référence à ce livre sera désormais indiquée par le sigle « CV » suivi de la pagination.

${ }^{4}$ Selon l'article de Sylvain Bissonnier, «Les réfugiés sur le Plateau, essai de typologie » dans Patrick Cabanel, Philippe Joutard, Jacques Sémelin, Annette Wieviorka (dir.), La Montagne refuge. Accueil et sauvetage des juifs autour du Chambon-sur-Lignon, Paris, Albin Michel, 2013, p. 82, les enfants de moins de seize ans, majoritairement juifs, représenteraient plus du quart des réfugiés $(27 \%)$.

${ }^{5}$ Ibid., p. 87.

6 Patrick Cabanel, Philippe Joutard, Jacques Sémelin, Annette Wieviorka, «Introduction générale », dans La Montagne refuge... op. cit., p. 9. En 1990, l'ensemble des habitants du village reçut le titre de Juste parmi les Nations de la part de 1'Institut israélien Yad Vashem, le titre est exceptionnellement attribué collectivement.
} 
Innocents de Philip Hallie en 1979, premier ouvrage sur l'histoire du Chambon, ou encore par Albert Camus, qui a passé quelques mois au village entre 1942 et $1943^{7}$. Toujours est-il que Gary a retenu l'histoire du Chambon. Sans doute, celui qui se dit né «d'un mélange de sang juif, cosaque et tartare $»^{8}$, a été d'autant plus sensible à l'action d'un village ayant sauvé des milliers de réfugiés ${ }^{9}$ condamnés à un sort dont n'ont pas réchappé certains membres de sa famille.

Le Chambon-sur-Lignon, pour le résumer d'après les termes des Cerfs-volants :

[...] était ce village qui, sous l'égide du pasteur André Trocmé, de sa femme Magda et avec l'aide de toute la population, avait sauvé de la déportation plusieurs centaines d'enfants juifs. Toute la vie du Chambon fut vouée pendant quatre ans à cette tâche. Et que j'écrive encore une fois ces noms de haute fidélité : Le Chambon-sur-Lignon et ses habitants [...]. (CV, 282)

L'humour comme la fiction se suspendent pour mettre en relief l'action admirable de ces hommes et femmes d'une " haute fidélité ». Chez un homme qui a combattu sa vie entière pour défendre l'idée que l'homme, malgré sa part d'inhumanité, n'est pas «une tentation impossible ${ }^{10}$, la «haute fidélité » semble être celle attachée à des valeurs comme «l'amour, la tendresse, la douceur, le pardon, le respect de la faiblesse » (CV 118). Tel l'engagement de Gary, celui des Justes du Chambon « venait beaucoup de... de ce que les gens croyaient encore en quelque chose ${ }^{11}$, les valeurs chrétiennes des Chambonnais étant hissées par l'auteur au rang plus vaste de l'engagement humain.

\footnotetext{
${ }^{7}$ C'est à la suite d'une rechute de tuberculose que Camus séjourne dans une ferme à proximité du Chambon. Il ne prend pas part à la résistance du Plateau mais a connaissance des actions de sauvetage entreprises dans la région. Pour cette question, voir l'article de Patrick Gérard Henry, «Albert Camus, le Plateau et la rédaction de La Peste » dans Patrick Cabanel, Philippe Joutard, Jacques Sémelin, Annette Wieviorka (dir.), La Montagne refuge..., op. cit., pp. 215-225.

${ }^{8}$ Romain Gary, La Promesse de l'aube [1960], Paris, Gallimard, coll. « folio », édition définitive, 1980, p. 283.

${ }^{9}$ Près de cinq mille réfugiés juifs auraient été recueillis par le Plateau des Cévennes d'après Alain Michel dans son article « 1941-1945. Les Justes des Nations et la Shoah», [en ligne]. URL : https://www.herodote.net/1941_1945-synthese- 74.php. Consulté le 20 août 2016

${ }^{10}$ Romain Gary, La Promesse de l'aube, op. cit., p. 283.

${ }^{11}$ Georgette Barraud, citation extraite du documentaire de Pierre Sauvage, Les Armes de l'esprit (1989), citée dans les « Portraits » du cahier central de Patrick Cabanel, Philippe Joutard, Jacques Sémelin, Annette Wieviorka (dir.), La Montagne refuge..., op. cit., page non précisée.
} 
C'est encore le nom de ce village qui résonne à la toute fin du roman, laissant entendre la voix de l'auteur qui se confond avec celle de Ludo : "Je termine enfin ce récit en écrivant encore une fois les noms du pasteur André Trocmé et celui de Le Chambon-sur-Lignon, car on ne saurait mieux dire » $(\mathrm{CV}, 369)$. Conclure en évoquant à nouveau l'action de ce village, c'est affirmer sans grandiloquence ni « idéalisme bêlant » ${ }^{12}$, et malgré des notes auparavant plus amères du narrateur sur « l'inhumanité » qui est « chose humaine » $(\mathrm{CV}, 265)$ l'espoir - timide, parfois volage, mais malgré tout têtu - que le totalitarisme aura toujours à en démordre face à "la résistance des faibles, l'amour en marche », selon la belle formule utilisée par Tzvetan Todorov pour parler des Justes du Chambon ${ }^{13}$.

\section{Écriture de la pudeur, écriture de la « juste mémoire »}

Le Chambon constitue donc également une sorte de village-refuge pour Romain Gary, à travers la démonstration de la persistance de certaines valeurs humaines par ses habitants. Pourtant, loin d'en faire le récit épique ou un éloge bien senti, la narration reste discrète dans son évocation du Chambon. Rappelons que Gary s'est toujours méfié des récits exaltés qui courent le risque selon lui d'«érige[r] l'Homme en valeur $\mathrm{H}$ mythologique et [de] fini[r] par rendre l'idée de l'homme plus précieuse que l'homme lui-même, ouvrant ainsi la porte à toutes les inhumanités $»^{14}$. La diégèse n'intègre pas directement l'action d'Ambroise Fleury quand il se rend sur le Plateau cévenol ni celle des Chambonnais : elles ne sont mentionnées qu'au détour des dialogues des personnages ou sur le mode de la citation par le narrateur. L'évocation feutrée de l'histoire du village semble alors restituer la discrétion et l'humilité de ses habitants qui, loin de glorifier leur action, estiment avoir réalisé la juste chose à faire en agissant simplement comme le montrent les témoignages recueillis par Pierre Sauvage dans son documentaire. Cette résistance

\footnotetext{
${ }^{12}$ Romain Gary ironise sur sa propension à l'idéalisme dans La nuit sera calme, [1974], Paris, Gallimard, coll. « folio », 1976, p. 85: «Je me connais très bien sociologiquement: je suis un bourgeois libéral à aspirations humanisantes et humanitaires, du genre Vendredi, hebdomadaire des années trente, je ne changerai jamais, et il s'agit toujours de moi lorsque l'extrême droite ou l'extrême gauche parlent d' 'idéalisme bêlant' ou d' 'humanisme bêlant' ".

${ }^{13}$ Tzvetan Todorov, Mémoire du mal, Tentation du bien : enquête sur le siècle, Paris, Robert Laffont, 2000, p. 244.

${ }^{14}$ Pour Sganarelle [1960], Paris, Gallimard, coll. « folio », édition définitive, 1980, p. 333.
} 
civile, selon les mots de Jacques Sémelin «n'a jamais fait de bruit. Elle n'a rien de spectaculaire. Elle ne mobilise aucune imagerie épique et opère dans la plus grande discrétion de l'accueil et de la protection. Ses armes sont le silence et l'invisibilité des chaînes de solidarité ${ }^{15}$. Précisons bien sûr que le mutisme des Chambonnais était de rigueur pour préserver la survie des réfugiés et l'efficacité de leur action de sauvetage face aux menaces de rafle.

L'écriture garyenne, par le mode de la citation ainsi que par la suspension de la parole lors de la dernière évocation du village, s'efforce alors de respecter le silence prudent de ces Justes de France. Le laconisme d'Ambroise Fleury, face à son neveu qui lui demande les raisons de son voyage, illustre d'ailleurs la discrétion des villageois :

- Sacré nom de nom, qu'est-ce que c'est que cette histoire ? Pourquoi Le Chambon ? Pourquoi les Cévennes?

Il sourit. Il y avait maintenant autant de rides sur son visage que de poils dans sa moustache.

- Parce qu'ils ont besoin de moi, là-bas. (CV, 281)

La retenue de l'écriture garyenne peut surtout se comprendre par une volonté d'éviter l'écueil du processus de commémoration qui peut «préserve[r] $»$ tout « en fossilisant ${ }^{16}$ selon Claudie Bernard. L'incipit du roman esquisse en effet une remise en question des processus officiels de commémoration, en s'ouvrant sur le sort réservé au musée des cerfsvolants fondé en hommage à l'oncle de Ludo. Le narrateur insiste sur l'inefficacité d'une politique mémorielle qui consiste à entretenir des lieux dédiés au passé, mais qui échoue à en raviver le souvenir, à l'instar de ce «petit musée consacré aux œuvres d'Ambroise Fleury, à Cléry » qui :

...n'est plus aujourd'hui qu'une attraction touristique mineure. [...] Malgré le peu d'intérêt qu'il suscite, et la modestie de la subvention qu'il reçoit de la municipalité, le musée ne risque pas de fermer ses portes, il est trop lié à notre histoire, mais la plupart du temps ses salles sont vides, car nous vivons une époque où les Français cherchent plutôt à oublier qu'à se souvenir. (CV, 9)

\footnotetext{
15 Jacques Sémelin, « Résistance civile : l'exemple emblématique du Chambon et de son Plateau » dans Patrick Cabanel, Philippe Joutard, Jacques Sémelin, Annette Wieviorka (dir.), La Montagne refuge..., op. cit., p. 72.

${ }^{16}$ Claudie Bernard, Le Passé recomposé. Le roman historique français du dix-neuvième siècle, Paris, Hachette supérieur, coll. « Recherches littéraires », 1996, p. 171.
} 
Pourtant, l'évocation répétée du nom du Chambon-sur-Lignon et de celui des époux Trocmé, souligne bien le désir garyen de préserver la mémoire du village :

Et que j'écrive encore une fois ces noms de haute fidélité : Le Chambon-surLignon et ses habitants, et s'il y a aujourd'hui oubli en la matière, que l'on sache que nous, les Fleury, nous avons toujours été des prodiges de la mémoire, et que je récite leurs noms souvent, sans en oublier un seul, puisqu'on dit que le cœur a besoin d'exercice. (CV, 282)

La voix de l'auteur se fait une nouvelle fois entendre à travers celle de Ludo. Comment, dès lors, éviter que l'oubli qui touche Ambroise Fleury, figure des Justes parmi les Nations dans le roman, se fasse également sur les habitants du Chambon? Quelle est la teneur de cet « exercice » mémoriel auquel se livre Gary pour répondre au défi d'une écriture de la $«$ juste mémoire $»^{17}$ ?

\section{Ambroise Fleury, figure palimpseste des Justes du Chambon-sur-Lignon?}

C'est au souhait de préserver le souvenir vivant du village que répond le personnage d'Ambroise Fleury, figure locale du village de Cléry, décrit comme un « objecteur de conscience » $(\mathrm{CV}, 32)$, comme un homme dont l'imaginaire enfantin se lit à travers les cerfs-volants qu'il fabrique. La sensibilité de ce « doux pacifique » $(\mathrm{CV}, 277)$ face au sort réservé aux juifs par le régime collaborationniste se lit dans sa réaction à la nouvelle de la rafle du Vel' d'Hiv :

- Tu as entendu la nouvelle, Ambroise ? La rafle du Vel' d'Hiv' ?

- Quelle rafle?

- Ils ont ramassé tous les Juifs et les ont déportés en Allemagne.

Mon oncle se taisait. Il n'y avait plus de cerf-volant auquel il aurait pu s'accrocher à ce moment-là. Duprat cogna du point sur la table.

- Et les enfants aussi, gronda-t-il. Ils ont livré les enfants aussi. On ne les reverra jamais vivants. Ambroise Fleury tenait un verre de vin à la main. Ce fut la seule fois dans ma vie où je vis sa main trembler. [...] Son visage était devenu gris et parut avoir deux fois plus de rides. (CV, 277)

\footnotetext{
${ }^{17}$ D'après la notion de Paul Ricœur, La Mémoire, l'Histoire, l'Oubli, Paris, Seuil, coll. «Points Essais », 2000, p. 1. Précisons que Paul Ricœur a lui-même enseigné la philosophie au collège Cévenol du Chambon-sur-Lignon de 1945 à 1948. Pendant la guerre, le collège accueillit des enfants juifs.
} 
La réaction physique de Fleury manifeste l'horreur qu'il éprouve en apprenant cet acte innommable. Incarcéré pour avoir fait voler « [s]ept cerfs-volants en forme d'étoiles juives » $(\mathrm{CV}, 279)$ en protestation à cette rafle, c'est en prison qu'il entend parler du Chambon-sur-Lignon et qu'il décide de s'y rendre.

André Trocmé a lui aussi réagi en protestation à la rafle du Vel' d'Hiv'. Lors de la visite de Georges Lamirand, secrétaire d'État à la Jeunesse du gouvernement de Vichy, il déclare: "Nous ignorons ce qu'est un juif, nous ne connaissons que des hommes $»^{18}$. On imagine bien Gary placer cette déclaration dans la bouche du facteur de Cléry. En effet, à travers le personnage haut en couleur d'Ambroise Fleury, la présence du Chambon-sur-Lignon dans le roman ne se limite pas au mode de la citation: c'est bel et bien «l'épaisseur humaine» des villageois que l'auteur semble vouloir restituer.

Certes, l'éthopée du personnage peut tout aussi bien s'inscrire, de façon plus vaste, dans un portrait représentatif des Justes parmi les Nations que l'on tenterait de dresser. Gérard Bollon, tout en rappelant qu'un tel exercice est fortement réducteur ${ }^{19}$, évoque certaines qualités comme «les convictions religieuses, la clairvoyance, la compassion, la rigueur éthique, la désobéissance civique pour raison de conscience » : autant de qualités qui, hormis la première, trouvent écho chez l'oncle Fleury. Cependant, certaines caractéristiques du personnage font plus précisément écho aux villageois du Chambon-sur-Lignon, et notamment à ses habitants les plus connus. Ainsi, tout comme Fleury qui ne perd pas une occasion de «manifester ses opinions pacifistes, défend[re] les objecteurs de conscience et condamn[er] toutes les formes de violence » $(\mathrm{CV}, 11)$, le Chambon apporte son soutien aux objecteurs de conscience et manifeste son engagement pacifique dès les années 1930 en signant par exemple une pétition demandant la limitation des armements. L'engagement pacifique du village se renforce à partir de 1934 avec l'arrivée du pasteur André Trocmé qui lui-même est objecteur de

\footnotetext{
${ }^{18}$ Cité par Jacques Sémelin, « Résistance civile : l'exemple emblématique du Chambon et de son Plateau », art. cité, p. 69.

${ }^{19}$ Gérard Bollon, « Des figures de sauveteurs « dans Patrick Cabanel, Philippe Joutard, Jacques Sémelin, Annette Wieviorka (dir.), La Montagne refuge..., op. cit., p. 143 : "Dresser un inventaire des traits de caractère pour cerner les comportements de ces hommes et de ces femmes reviendrait à les enfermer dans une sorte de déterminisme qui nierait la responsabilité individuelle de chacun [...] essayer de systématiser la compréhension des raisons aboutit certainement à ignorer bien des vides dans les histoires individuelles sans répondre au pourquoi ».
} 
conscience $^{20}$. Cet engagement s'illustre parfaitement dans le sermon que prononce le pasteur le 23 juin 1940, appelant les villageois à résister par les « armes de l'Esprit»:

[...] Des pressions païennes formidables vont s'exercer sur nous-mêmes et sur nos familles, pour tenter de nous entraîner à une soumission passive à l'idéologie totalitaire. Si l'on ne parvient pas tout de suite à soumettre nos âmes, on voudra soumettre tout au moins nos corps. Le devoir des chrétiens est d'opposer à la violence exercée sur leur conscience les armes de l'Esprit. [... $]^{21}$

Si Gary écarte l'influence religieuse de l'engagement dans la Résistance des Cerfs-volants, la résistance spirituelle d'Ambroise Fleury a en quelque sorte toujours partie liée avec le ciel. Les cerfs-volants qu'il fabrique incarnent parfaitement cette «lutte par les armes de l'esprit» formulée par André Trocmé. Parmi les hommes dont il persiste à faire voler les effigies dans le ciel malgré l'interdiction des nazis, on trouve entre autres Rabelais, Montaigne, Rousseau, Victor Hugo, Jean Jaurès : des humanistes qui, en leur temps, ont lutté à travers leurs œuvres et leurs actes pour la fraternité et la liberté, qui ont combattu toutes les formes de cette "Puissance de la réalité ${ }^{22}$ conceptualisée par Gary. Jusque dans les camps de concentration et d'extermination, l'art des cerfs-volants d'Ambroise Fleury se veut une protestation contre le totalitarisme. À Buchenwald, alors que « beaucoup ne tenaient à la vie que par ce qui n'a pas de corps », Fleury apporte de l'espoir aux concentrationnaires et continue de créer des « cerfs-volants aux couleurs gaies qui semblaient proclamer [son] espoir et [s]a confiance impérissables » et persiste même à «donner à certaines de ses œuvres les traits de Rabelais et de Montaigne » (CV, 319). S'il est déporté à Auschwitz, c'est parce qu'il persiste à rejeter toute " soumission passive à l'idéologie totalitaire », en

\footnotetext{
${ }^{20}$ Ces informations sont tirées de l'article de François Boulet, « Les protestants de la Montagne dans l'entre-deux- guerres » dans Patrick Cabanel, Philippe Joutard, Jacques Sémelin, Annette Wieviorka (dir.), La Montagne refuge ..., op. cit., pp. 25-37.

${ }^{21}$ Cité par Alain Michel, «1941-1945. Les Justes des Nations et la Shoah», art. cité. « Les armes de l'esprit » est aussi le titre d'un ouvrage de l'historienne Renée Bédarida sur le journal clandestin Témoignage chrétien. Toute jeune encore, elle s'engagea dans la Résistance au sein du mouvement qui diffusait le journal. Renée Bédarida, Les Armes de l'Esprit. Témoignage chrétien (1941-1944), Paris, éditions ouvrières, 1977.

${ }^{22}$ Dans Pour Sganarelle, Romain Gary introduit le concept de «Puissance de la réalité ». Cette notion désigne l'idéologie totalitaire et englobe plus largement tout ce qui rappelle l'homme à sa fragilité, oppose à sa liberté des déterminismes, et le convainc de son impuissance à s'extraire de sa condition.
} 
refusant la demande d'Ilse $\mathrm{Koch}^{23}$ gardienne au camp de femmes de Buchenwald, de lui fabriquer un cerf-volant en peau humaine.

On ne peut pas bien sûr prétendre que Gary ait eu une connaissance approfondie du Chambon, de la vie et du caractère de ses habitants. Peutêtre s'est-il essayé à recréer l'idée qu'il se faisait de la personnalité des Justes du village. Toujours est-il que les biographèmes de certaines figures du Chambon font parfois - volontairement ou fortuitement - écho à certains traits d'Ambroise Fleury. Ainsi, l'imaginaire enfantin ${ }^{24}$ de ce dernier et sa connaissance de l'histoire qui se manifestent à travers ses cerfs-volants, sa foi en l'enseignement public obligatoire, son attachement à Léon Blum ${ }^{25}$, rappellent par exemple Roger Darcissac, que Gérard Bollon décrit comme un passionné d'histoire, dessinateur horspair, membre de la SFIO et secrétaire d'une société pacifiste, La Fraternelle $^{26}$. Ce directeur du collège Cévenol protège ses élèves juifs en cachant leur origine. Quant à André Trocmé, homme «à la très forte personnalité ${ }^{27}$, tout comme Fleury avec ses cerfs-volants, il met l'imaginaire au service de la lutte contre le nazisme en créant des spectacles, des pièces de théâtre ou encore des contes pour enfants avec le recueil L'Église de neige, notamment illustré à l'aide de grandes ombres chinoises par Roger Darcissac lors d'un Noël sous le régime de Vichy. Le pasteur Trocmé est de plus à l'origine de la création du collège Cévenol en 1938, collège qui permet aux jeunes élèves de la population locale de poursuivre leurs études secondaires. Ainsi, par les divers traits que Gary insuffle à son personnage, Ambroise Fleury semble bien être l'incarnation fictive de l'état d'esprit du Chambon-sur-Lignon, voire une figure palimpseste établie à partir des portraits des Justes du village. Raccrochons-nous enfin à une précision onomastique: le nom de «Fleury », s'il rappelle le patronyme de Jean Fleury, premier Juste de

\footnotetext{
${ }^{23}$ La fiction recoupe ici l'Histoire en citant le nom d'Ilse Koch (1906-1967), réelle gardienne au camp de Buchenwald.

${ }^{24}$ Parmi les cerfs-volants qu'il crée, citons entres autres « Titube, une sorte de grenouille hilare, avec des pattes qui faisaient 'bonjour' dans le vent, [...] Clapote, lequel était un poisson tout souriant, frémissant dans les airs de ses écailles argentées et de ses nageoires roses, [...] Mimile, un martien, que je trouvais bien gentil, avec ses yeux ronds et des ailes en forme d'oreilles qui se mettaient à frétiller quand il s'élevait » ou encore « Croquemuche, Batifol, Clopin-clopant, Patapouf [...]» (CV, 12).

${ }^{25}$ Ambroise Fleury fabrique « un Léon Blum en papier, ficelle, carton et queue orientale, lequel avait belle allure dans le ciel, avec son chapeau noir et ses bras levés avec éloquence » $(\mathrm{CV}, 76)$.

${ }^{26}$ Gérard Bollon, « Des figures de sauveteurs », art. cité, pp. 148-149.

${ }^{27}$ Les informations citées sur André Trocmé sont extraites de l'article de François Boulet, «Les protestants de la Montagne dans l'entre-deux-guerres », art. cité, p. 32.
} 
France, comme le remarque Kerwin Spire ${ }^{28}$, fait de plus écho au «Coteau fleuri» de la Cimade $^{29}$, l'un des centres d'accueil pour les réfugiés juifs du Chambon.

\section{Le retour des camps d'Ambroise Fleury ou la victoire de " tout ce qui demeure éternellement pur et inaltérable sur cette terre »}

Jacques Lecarme voit en André Trocmé « le seul vrai vainqueur de cette guerre, et le seul personnage parfaitement humain de cette histoire, mi fictive-mi vraie ${ }^{30}$. Quid du pendant fictif d'André Trocmé, Ambroise Fleury? L'oncle de Ludo rentre, en effet, non seulement vivant mais les moustaches intactes des camps de la mort:

Il n'avait pas changé. Il n'avait pas vieilli. Sa moustache était tout aussi longue et tout aussi épaisse et l'œil tout aussi sombre dans la gaieté. Ils n'y peuvent rien. Je ne sais pas ce que j'entendais par « ils ». Les nazis, peut-être, ou simplement tous ceux qui. $(\mathrm{CV}, 367)$

La remarque finale de Ludo montre bien que le retour miraculeux de Fleury symbolise une forme de victoire sur le totalitarisme. La fiction déjoue ici les règles du réel ${ }^{31}$ pour conclure sur une note clairement optimiste. Celui qui se dit «condamné au happy end» dans Pour Sganarelle, en explicite la raison : « [1]e Roman ne peut pas finir mal. [...] dès qu'il n'y a pas d'espoir, il n'y a pas de Roman total, pas de rivalité possible avec la Puissance, il n'y a plus que soumission totalitaire $[\ldots] »^{32}$.

\footnotetext{
${ }^{28}$ Kerwin Spire, « Romain Gary, des Compagnons de la Libération aux Justes parmi les nations », art. cité, p. 82. L'abbé Jean Fleury obtint le titre de Juste parmi les Nations en 1964 pour son action de sauvetage de juifs dans la région de Poitiers. Il aida aussi des Tsiganes.

${ }^{29}$ La Cimade, créée en 1939 (Comité inter-mouvement auprès des évacués), venait en aide aux populations protestantes alsaciennes transplantées dans le Limousin. Après la défaite française, elle vient en aide aux réfugiés juifs en les accueillant, légalement puis clandestinement à partir d'août 1942, au Coteau fleuri.

${ }^{30}$ Jacques Lecarme, «Relire Éducation européenne (1945) à travers Les Cerfs-volants (1980) », Roman 20-50, $\mathrm{n}^{\circ}$ 32, déc. 2001, Romain Gary - Émile Ajar, Éducation européenne et La Vie devant soi, p. 66.

${ }^{31}$ Dans l'histoire du Chambon-sur-Lignon, le sort des déportés est loin d'être comparable à celui de Fleury. Daniel Trocmé, cousin d'André Trocmé, est arrêté le 29 juin 1943 à la Maison des roches, un internat du collège Cévenol, avec dix-huit de ses élèves. Déporté à Buchenwald, puis à Dora, il meurt dans le camp de Maidanek en Pologne. Les étudiants sont déportés à Buchenwald et Auschwitz. Ils seront sept à en revenir, les six étudiants juifs meurent en déportation.

${ }^{32}$ Romain Gary, Pour Sganarelle, op. cit., p. 128.
} 
Dès lors, « [s]i la réalité porte avant tout la marque du malheur, le roman [...] ne peut manquer de saisir cette chance de rivaliser victorieusement avec la Puissance par la création d'un monde romanesque éclairé par le rire et l'espoir » ${ }^{33}$, portés ici par le « facteur timbré » $(\mathrm{CV}, 10)$ des Cerfsvolants.

Dans son dernier roman, les ultima verba de Gary gardent la trace d'une lutte avec la Puissance : lutte d'une irrévérence savoureuse avec le défi au réel incarné par le retour de Fleury, lutte résolument réaliste par l'exemple des époux Trocmé et du Chambon-sur-Lignon. En reprenant les mots qu'emploie Madame Julie - maquerelle juive qui résiste à sa manière dans le roman - pour qualifier les cerfs-volants de Fleury, on pourrait dire que la Résistance du pasteur du Chambon, comme celle du facteur de Cléry, incarnent «tout ce qui demeure éternellement pur et inaltérable sur cette terre » $(\mathrm{CV}, 363)$.

${ }^{33}$ Ibid., p. 361. 